\title{
Trypanosoma brucei Infection in a HIV Positive Ugandan Male
}

\author{
ELIZABETH A DILL, CYBELE RENAULT, BETH D KIRKPATRICK
}

\begin{abstract}
Human African Trypanosomiasis, or African Sleeping Sickness, is a parasitic infection caused by Trypanosoma brucei (gambiense or rhodesiense), and one of the declared neglected tropical diseases. ${ }^{1}$ Sleeping sickness has high fatality rates and is a continued threat in several African countries. ${ }^{2}$ We present characteristic clinical and microbiologic features of a fatal case of African Sleeping Sickness in an HIV-infected individual.
\end{abstract}

INDEX TERMS: African sleeping sickness; Trypanosomiasis, African; Nagana

ABBREVIATIONS: HAT - Human African Trypanosomiasis, CNS - central nervous system, CSF cerebrospinal fluid, T/S - trimethoprim/sulfamethoxazole, HIV - Human Immunodeficiency Virus, bpm - beats per minute, AFB - Acid fast bacilli, hpf - high powered field, lpf - low powered field.

Clin Lab Sci 2011;24(2):85

Elizabeth A. Dill, University of Vermont, Burlington, $V T$.

Cybele Renault, MD, Standford Universtiy, Stanford, $C A$.

Beth D. Kirkpatrick, MD., University of Vermont, Burlington, VT.

Address for Correspondence: Elizabeth A. Dill, University of Vermont, 95 Carrigan Drive, Burlington, VT 05405, 802-656-0016, Elizabeth.Dill@uvm.edu

\section{INTRODUCTION}

Human African Trypanosomiasis (HAT) is a major threat to approximately 60 million people living in 36 countries in sub-Saharan Africa. ${ }^{2,3}$ There are two forms of illness: a more chronic infection caused by the protozoan parasite Trypanosoma brucei rhodesiense (West African) and a more acute disease caused by Trypanosoma brucei gambiense (East African). ${ }^{3}$ Greater than twenty species of Glossina tsetse flies are capable of transmitting the parasites; humans and animals act as reservoirs. ${ }^{1,3}$ Common symptoms of the early disease include but are not limited to headache, fever, malaise, and arthralgia. ${ }^{1}$ The second phase of disease is referred to as "Sleeping Sickness" as trypanosomes invade the central nervous system (CNS). ${ }^{3}$ Patients in advanced stages experience daytime lethargy and nighttime insomnia as well as symptoms of meningo-encephalitis. If left untreated, trypanosomiasis in the second (CNS) phase is always fatal. ${ }^{1}$ We present a case of HAT in a male infected with the human immunodeficiency virus (HIV).

\section{CASE PRESENTATION}

A 42 year old HIV positive male from the West Nile region of Uganda presented to a local hospital with nausea and vomiting after eating, as well as fever occurring in the evenings. His fever had been present for two weeks, and began one week after leaving Western Uganda. The patient also had an intermittent, productive cough. He denied arthralgias, myalgias and all central nervous system (CNS) symptoms including headache, shaking and chills.

The patient's last known absolute CD4 count was $298 / \mu \mathrm{L}(702-1791 / \mu \mathrm{L})$, however the date of the count was unknown. $\mathrm{He}$ was on trimethoprim/sulfamethoxazole (T/S) prophylaxis and had no known drug allergies. There was no known history of opportunistic infections, including tuberculosis. The patient was married with four children, had a history of alcohol use/abuse and was an ex-smoker. 


\section{CLINICAL PRACTICE}

Upon admission to the emergency department, his temperature was $36.7^{\circ} \mathrm{C}$, heart rate was 100 beats per minute (bpm), and his blood pressure was 90/60. The clinician noted coarse crackles in right lung; no lymphadenopathy was present. Initial differential diagnosis included tuberculosis, pneumonia, Pneumocystis jivoveci pneumonia and sepsis. He was given empiric ceftriaxone and quinine. A sputum sample was sent to the laboratory for an acid fast bacilli (AFB) culture; thick and thin malaria smears were collected.

On day 1, the patient had no complaints, was afebrile (temperature $37^{\circ} \mathrm{C}$ ) and his blood pressure was $130 / 72$. Beginning day 2 , the patient had a fever of $39^{\circ} \mathrm{C}$, heart rate of $84 \mathrm{bpm}$, a respiratory rate of 32 breaths per min, and his blood pressure was 110/70. The malaria smear from peripheral blood was confirmed negative (thick and thin prep as well as wet mount). However the wet mount smear demonstrated 8-10 trypanosomes per high powered field (hpf), the thick prep showed between 0 10 organisms per hpf (see figure 1), and 0-1 per low powered field (lpf). Because it was not immediately available at the treating hospital, the therapeutic antiprotozoal agent Melarsoprol (use is described below) was ordered from an outlying area.

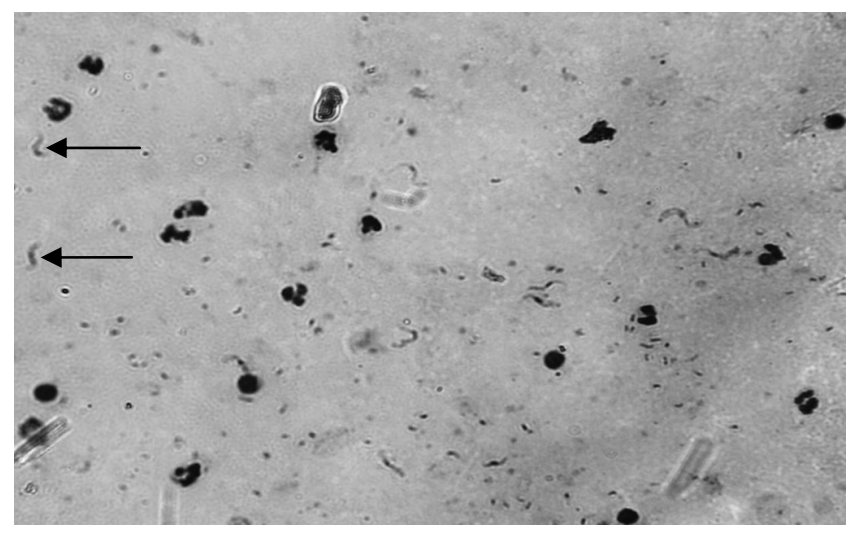

Figure 1: Peripheral thick blood smear demonstrating Trypanosome parasites. Geimsa stained thick blood smear (40X magnification) demonstrating trypomastigotes of $T$. b.gambiense or rhodesiense (morphologically indistinguishable). Parasites have a small kinetoplast and an undulating membrane.

The patient began to experience insomnia and hiccups on days 4 and 5 . On day 6, a lumbar puncture was performed. Results of the lumbar puncture showed clear, colorless cerebrospinal fluid (CSF), with normal protein levels, however his glucose was low. Trypanosomes were seen in the CSF microscopically (0$1 / \mathrm{hpf}$ ). On day 7 , new onset of myalgias and arthralgias occurred. During day 8, the patient experienced severe headache, nausea and vomiting and died on day 9. Autopsy was not performed.

\section{DISCUSSION}

There are two types of African Sleeping Sickness/ Human African Trypanosomiasis (HAT), which cause distinct clinical features. HAT in West and Central Africa is typically a chronic infection caused by the protozoan parasite Trypanosoma brucei gambiense while Trypanosoma brucei rhodesiense causes a more acute form of the illness and is most often found in East Africa. ${ }^{2,4}$ There are currently about 50-70,000 cases of trypanosomiasis in sub-Saharan Africa with about $90 \%$ of cases being more chronic infections caused by Trypanosoma brucei gambiense. ${ }^{2,5}$ Both forms of HAT are present in Uganda. ${ }^{5}$ No data exists to determine whether HIV-infection increases risk or disease progression.

There are two stages of HAT; the hemolymphatic stage (the early stage) and encephalitic/ CNS stage (the second, late stage). The timeframe of transition from the first stage to the second occurs more rapidly in those infected with Rhodesian sleeping sickness. ${ }^{3,4}$ Approximately 5-15 days post-infection, a trypanosomal chancre, caused by the accumulation of trypanosomes in the connective tissue, may form at the site of the tsetse fly bite. ${ }^{3,6}$ The parasites spread through the lymphatics and bloodstream about 1-3 weeks after the bite and invade the lymph nodes, liver, spleen, heart, endocrine system, and eyes; this is the hemolymphatic stage. $^{3}$ During this period, infected individuals may also experience non-specific symptoms such as headache, fever, nausea and vomiting, weakness, sweating, and arthralgia. Waterbottom's sign may also be seen; an abnormality in which the patient's posterior cervical lymph nodes become enlarged. ${ }^{3}$

If a person remains untreated, the parasites will eventually cross the blood-brain barrier and enter the CSF; this is the encephalitic or second stage. ${ }^{3}$ It is at this 


\section{CLINICAL PRACTICE}

stage that neurologic symptoms are observed and the sleep cycle may be disrupted, causing patients to experience insomnia during the night and slumber during daytime hours. The hiccups experienced by this patient were likely due to CNS infection and subsequent release of inhibition of the normal hiccup reflex. Also during this second stage, as part of a meningo-encephalitis, the meninges thicken and are infiltrated by lymphocytes, plasma cells and mott cells (abnormal plasma cells formed when immunoglobulin accumulates in Russell bodies instead of being secreted). ${ }^{6,7}$

In Gambian sleeping sickness, patients can be infected for months to years without noticing symptoms, however when symptoms emerge, the patient is typically in the advanced disease stage. ${ }^{2}$ In more acute infections (Rhodesian sleeping sickness), development of the first CNS symptoms takes weeks to months, and progression from systemic infection to CNS invasion occurs more rapidly. ${ }^{2,3}$

\section{Diagnosis:}

Definite diagnosis is made when the characteristic trypanosoma parasite (trypomastigote form) is observed in blood, lymph nodes, scrapings from the chancre, bone marrow, or CSF. ${ }^{1,4}$ Typically, thick and thin smears are made using whole blood or buffy coat and are Giemsa or Wright stained in order to aid in observation of the parasite. ${ }^{8}$ Live, motile trypanosomes may be seen in fresh wet mount preparations of blood or buffy coat samples. ${ }^{1,8}$ The trypanosomes responsible for HAT are indistinguishable from other trypanosomal infections, including T. cruzi (Chagas' disease). As in our case, the distinction between the species is difficult in resource poor areas. A card agglutination test is available for Trypanosoma brucei gambiense (CATT) and is used as a screening tool in parts of Africa. ${ }^{1,8}$ Presence of trypanosomes in the CSF allows providers to distinguish between the early and late stages of illness, and presence of mott cells in CSF are highly indicative of HAT. ${ }^{7,8}$ Diagnosis during the encephalitic stage may be challenging due to sparseness of organism, and a non-invasive test capable of distinguishing early from late stage disease has not been developed. ${ }^{3}$

\section{Treatment:}

The drug of choice for treating the hemolymphatic stage of Gambian sleeping sickness is pentamidine isethionate as it has a cure rate of $93-98 \% .^{1}$ It is administered intramuscularly due to increased risk of hypotension after intravenous administration, and the typical dosing schedule is $4 \mathrm{mg} / \mathrm{kg}$ for seven days. ${ }^{1}$ It is less effective than suramin against Trypanosoma brucei rhodensiense and is also an ineffective treatment for the encephalitic stage of trypanosomiasis. ${ }^{3,6}$ The drug is typically tolerated well, although minor adverse drug reactions are common. Immediate adverse reactions may be seen including hypotension which occurs in about $10 \%$ of patients who receive treatment via intramuscular injection and in up to $75 \%$ of patients who receive treatment intravenously. ${ }^{1}$

Suramin is an alternative treatment for pentamidine and can be used to treat both Gambian and Rhodesian forms of sleeping sickness. Because it is incapable of crossing the blood brain barrier, it is not a treatment for patients in the second stage of illness. ${ }^{6}$ The drug is typically administered by injecting about $4-5 \mathrm{mg} / \mathrm{kg}$ body weight intravenously on the first day, followed by five injections of $20 \mathrm{mg} / \mathrm{kg}$ body weight over approximately one month (one injection every seven days). ${ }^{1}$ Due to the toxicity of suramin, renal damage may occur, however the damage is usually mild and reversible. ${ }^{1,3,6}$

The treatment of choice for the encephalitic stage is melarsoprol; it is administered intravenously in various doses (dependent upon the case). Typically, three to four doses are given every 24 hours over 7-10 days. ${ }^{1,3,6}$ The major adverse reaction caused by melarsoprol occurs in about $5-10 \%$ of treated cases and is called encephalopathic syndrome; a syndrome which is fatal for $10-70 \%$ of patients. ${ }^{1}$

An alternative drug for the encephalitic stage of Gambian sleeping sickness is eflornithine. ${ }^{1}$ It is most often given at a dosage of $100 \mathrm{mg} / \mathrm{kg}$ body weight intravenously every 6 hours over a span of 14 days. ${ }^{1}$ Adverse reactions to eflornithine occur fairly frequently; about $25-50 \%$ of patients receiving eflornithine treatment will experience bone marrow toxicity and 


\section{CLINICAL PRACTICE}

roughly $10 \%$ of patients experience gastrointestinal symptoms. ${ }^{1}$

Based on new information from the WHO, it appears these therapy options will soon be replaced with a Nifurtimox-Eflornithine combination treatment regimen, which is easier to administer and has a shorter treatment duration than either melarsoprol or enflornithine alone. ${ }^{9}$

Treated patients should undergo lumbar puncture, blood analysis and regular follow-up examinations, every 6 months, for at least two years following treatment to ensure infection clearance. ${ }^{1,3,6}$

\section{CONCLUSION}

There is a continued threat of HAT in Africa, particularly in Uganda, Malawi and the United Republic of Tanzania. Because HAT is fatal in the secondary phase if left untreated, and safe, effective treatment options are not available, more resources should be allocated to research and therapeutic agent development in an effort to improve early diagnostics and treatment of this neglected disease. Due to the scarcity of this disease in the United States, it is important for laboratory professionals to recognize the diagnostic characteristics of this disease.

\section{REFERENCES}

1. Cook GC, Zumla A, Manson P. Manson's tropical diseases. 22nd ed. [Edinburgh]: Saunders; 2009.

2. African trypanosomiasis (sleeping sickness). (Accessed April 5, 2010, at http://www.who.int/mediacentre/factsheets/fs259/en/)

3. Kennedy PG. The continuing problem of human African trypanosomiasis (sleeping sickness). Ann Neurol 2008;64:11626.

4. Kager PA, Schipper HG, Stam J, Majoie CB. Magnetic resonance imaging findings in human African trypanosomiasis: a four-year follow-up study in a patient and review of the literature. Am J Trop Med Hyg 2009;80:947-52.

5. Hotez PJ, Kamath A. Neglected tropical diseases in sub-saharan Africa: review of their prevalence, distribution, and disease burden. PLoS Negl Trop Dis 2009;3:e412.

6. Cecil RL, Goldman L, Ausiello DA. Cecil textbook of medicine. 22nd ed. Philadelphia, Pa.: Saunders; 2004.

7. Tarlinton D, Forster I, Rajewsky K. An explanation for the defect in secretion of IgM Mott cells and their predominant occurrence in the Ly-1 B cell compartment. Eur J Immunol 1992;22:531-9.

8. Rosenblatt JE. Laboratory diagnosis of infections due to blood and tissue parasites. Clin Infect Dis 2009;49:1103-8.

9. Nifurtimox-eflornithine combination treatment for sleeping sickness (human African trypanosomiasis): WHO wraps up training of key health care personnel. (Accessed April 16, 2010, at http://www.who.int/trypanosomiasis_african/research/combi nation_treatment/en/index.html. 